\title{
New prospects for polarized hyperon fragmentation functions
}

\author{
J. Soffer ${ }^{\mathrm{a}}$ \\ ${ }^{a}$ Centre de Physique Théorique, CNRS Luminy Case 907, \\ F 13288 Marseille Cedex 09, France
}

\begin{abstract}
We study the inclusive production of $\Lambda(\bar{\Lambda})$ in several high energy collision processes $\left(e^{+} e^{-}, e^{ \pm} p, \nu(\bar{\nu}) p, p p\right)$, in view of an accurate determination of the unpolarized and polarized fragmentation functions of a quark into a $\Lambda(\bar{\Lambda})$. For polarized fragmentation functions the inaccuracy and the scarcity of present data do not allow to distinguish between various theoretical models. We will indicate how future measurements will provide ways to discriminate between them and also how to achieve a necessary quark flavor separation. A possible extension to the production of the other hyperons $\left(\Sigma^{ \pm, 0}, \Xi^{-, 0}\right)$, will be also briefly discussed.
\end{abstract}

\section{Introduction}

The usual parton $(f=q, \bar{q}, g)$ distribution functions $f_{H}\left(x, Q^{2}\right)$ of a hadron $H$, which are extracted from Deep Inelastic Scattering (DIS) for a space-like energy scale $\left(Q^{2} \leq 0\right)$, have counterparts in the time-like region $\left(Q^{2} \geq 0\right)$, which are the hadron fragmentation functions $D_{f}^{H}\left(z, Q^{2}\right)$. They represent the probability to find the hadron $H$ with a fraction $z$ of the momentum of the parent parton $f$, at a given value of $Q^{2}$. Like distribution functions, fragmentation functions are universal, that is process-independent, and likewise, their $Q^{2}$ dependence is fully predicted by the QCD evolution equations. They can be measured in various high energy collision processes namely, $e^{+} e^{-}$annihilation, $e^{+} e^{-} \rightarrow\left(\gamma^{*}, Z\right) \rightarrow$ $H X$, semi-inclusive DIS, $l p \rightarrow l^{\prime} H X$ and singleinclusive $p p$ collisions, $p p \rightarrow H X$. So far, the best known fragmentation funtions are $D_{f}^{\pi}$ and $D_{f}^{K}$, corresponding to the most copiously produced light mesons $\pi$ and $K$. Our purpose here is to consider the hyperon fragmentation functions and more specifically to examine the case of $\Lambda(\bar{\Lambda})$. The self-analyzing properties of $\Lambda(\bar{\Lambda})$ make this spin-half baryon particularly appealing, to study spin transfer mechanisms. We first recall the results of a $\mathrm{QCD}$ analysis of the data for inclusive $(\Lambda+\bar{\Lambda})$ production in $e^{+} e^{-}$collisions, which yields the first simple and reliable parametrization of the unpolarized fragmentation functions $D_{f}^{\Lambda, \bar{\Lambda}}\left(z, Q^{2}\right)$. The observed longitudinal polarization of the $\Lambda$ 's produced at LEP on the $Z$-resonance, leads to some inaccurate information on the spin-dependent fragmentation functions $\Delta D_{f}^{\Lambda}\left(z, Q^{2}\right)$. As we will see, several theoretical models have been proposed for these polarized fragmentation functions which are, so far, badly constrained by existing data. Some predictions can be made for the spin transfer in polarized DIS, but one gets no definite conclusion by comparing them with the present very poor data from HERMES at DESY and E665 at FNAL. We also stress the importance of the $\Lambda(\bar{\Lambda})$ production in neutrino (antineutrino) DIS, which allows a clean flavor and spin separation. We will show some new data from NOMAD at CERN and comment on future experimental possibilities.

We will also give the prospects from $p p$ collisions with polarized protons at RHIC-BNL, because there are recent interesting suggestions for measuring the helicity (and transversity) transfer asymmetry in the process $p \vec{p} \rightarrow \vec{\Lambda} X$. From its dependence on the rapidity of the $\Lambda$, it is possible to discriminate easily between the various theoretical models, thanks to the high luminosity and the small statistical errors. Of course, this can be easely extended to the production of the other hyperons $\left(\Sigma^{ \pm, 0}, \Xi^{-, 0}\right)$, which will be also briefly discussed. 


\section{2. $e^{+} e^{-}$collisions}

From the production of $\Lambda(\bar{\Lambda})$ in $e^{+} e^{-}$annihilation one can determine $D_{f}^{\Lambda}\left(z, Q^{2}\right)$ by using a standard parametrization, at initial scale $\mu^{2}$

$D_{f}^{\Lambda}\left(z, \mu^{2}\right)=N_{f} z^{\alpha_{f}}(1-z)^{\beta_{f}}$.

To reduce the number of free parameters occuring above, one must make some simplifying assumptions based on, e.g. $S U_{f}(3)$ arguments. Ten free parameters, needed to include light quarks, heavy quarks and gluons, were obtained by fitting the world data for $14 \mathrm{GeV} \leq \sqrt{s} \leq 91.2 \mathrm{GeV}$, in a leading and next-to-leading order (LO and NLO) QCD analysis [1]. Although, it leads to a good determination of $D_{f}^{\Lambda}$, there is no flavor separation for the dominant contributions $D_{q}^{\Lambda}(q=u, d, s)$ and no separation between $D_{q}^{\Lambda}$ and $D_{\bar{q}}^{\Lambda}$, since the data does not separate $\Lambda$ and $(\bar{\Lambda})$. Other sets of quark fragmentation functions into $\Lambda(\bar{\Lambda})$, based on a bag model calculation and a fit to $e^{+} e^{-}$data, have been published recently [2].

The fragmentation of a longitudinally polarized parton into a longitudinally polarized $\Lambda$ will be described in terms of

$\Delta D_{f}^{\Lambda}\left(z, Q^{2}\right)=D_{f(+)}^{\Lambda(+)}\left(z, Q^{2}\right)-D_{f(+)}^{\Lambda(-)}\left(z, Q^{2}\right)$,

where $D_{f(+)}^{\Lambda(+)}\left(z, Q^{2}\right)\left[D_{f(+)}^{\Lambda(-)}\left(z, Q^{2}\right)\right]$ is the probability to find a $\Lambda$ with positive [negative] helicity in a parton $f$ with positive helicity. Clearly one obtains the unpolarized fragmentation function $D_{f}^{\Lambda}$ by taking in (2), the sum instead of the difference. For the fragmentation of a transversely polarized parton into a transversely polarized $\Lambda$, one uses (2) to define $\Delta_{T} D_{f}^{\Lambda}\left(z, Q^{2}\right)$ in complete analogy with $\Delta D_{f}^{\Lambda}\left(z, Q^{2}\right)$.

In order to construct $\Delta D_{f}^{\Lambda}$, one usually makes the assumption

$\Delta D_{f}^{\Lambda}(z)=C_{f}^{\Lambda}(z) D_{f}^{\Lambda}(z)$,

at a given scale, where $C_{f}^{\Lambda}(z)$ are the spin transfer coefficients, for which several models can be found in the literature. In Ref.[1], $\Delta D_{f}^{\Lambda}$ was neglected for heavy quarks and gluon and they take the following simple parametrization

$\Delta D_{u}^{\Lambda}=\Delta D_{d}^{\Lambda}=N_{u} \Delta D_{s}^{\Lambda}$ and $\Delta D_{s}^{\Lambda}=z^{\alpha} D_{s},(4)$

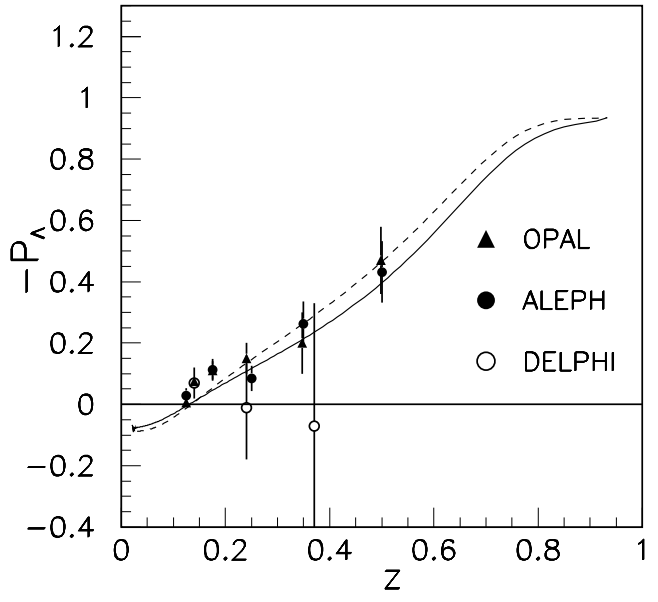

Figure 1. Comparison of the LEP data for $\left(-P_{\Lambda}\right)$, at the $Z$-pole, with the theoretical calculations of the $\mathrm{SU}(6)$ quark-diquark model (Taken from Ref.[p]).

in terms of two new parameters $\alpha$ and $N_{u}$ at a given scale. To cover a wide range of plausible models, they consider three different scenarios:

1) In the non-relativistic quark model, only strange quarks can contribute, therefore $N_{u}=0$. 2) By transposing the estimate of Ref. [3] for the polarized $u$ and $d$ quark distributions in a $\Lambda$, to the polarized fragmentation functions, one gets $N_{u}=-0.2$.

3) By assuming that all polarized fragmentation functions are equal, which is an extreme case, one gets $N_{u}=1$.

In Ref.[2], only two scenarios similar to 1) and 2), were considered and for other alternatives along the same lines, see also Ref. [4. A different way to construct $\Delta D_{q}^{\Lambda}(z)$ was proposed in Ref. [5], based on our knowledge of the quark distributions and by means of the simple reciprocity relation, the so called Gribov-Lipatov relation [6]

$D_{q}^{H}(z) \sim q_{H}(x)$,

where $q_{H}(x)$ is the quark distribution for finding the quark $q$ carrying a momentum fraction $x=z$ inside the hadron $H$.

The longitudinal $\Lambda$ polarization $P_{\Lambda}$, which can 
be directly expressed in terms of the $\Delta D_{f}^{\Lambda}$ 's (see ref.(1]), has been measured at LEP by several experiments and is shown in Fig.1. It is large and negative and consistent with the SU(6) quarkdiquark model proposed in Ref.[5], as well as scenario 3) from Ref.[1], mentioned above. In a very recent work [7], one finds various predictions for $P_{B}$, where $B$ stands for any hyperon of the octet baryon, obtained by using SU(3) symmetry relations.

\section{Charged lepton DIS process}

These results can be used to make predictions for $\Lambda$ production in semi-inclusive DIS, with only the electron (muon) beam longitudinally polarized. The $\Lambda$ polarization $P_{\Lambda}$ along its own momentum is related ( for the exact expression see Ref. [8]) to the longitudinal spin transfer $A^{\Lambda}(x, z)$ defined as

$A^{\Lambda}(x, z)=\frac{\sum_{q} e_{q}^{2} q^{N}(x) \Delta D_{q}^{\Lambda}(z)+(q \rightarrow \bar{q})}{\sum_{q} e_{q}^{2} q^{N}(x) D_{q}^{\Lambda}(z)+(q \rightarrow \bar{q})}$.

From the above values of $N_{u}$, one anticipates that $A^{\Lambda}$ will be small for 1), negative for 2) and large and positive for 3 ) and the results can be found in Ref.[1]. Another set of predictions is given in Ref. [4] and it will be interesting to compare them with data from future polarized DIS experiments, in particular from HERMES at DESY and COMPASS at CERN.

For the time being, we only have the few data points displayed in Fig.2 for the spin transfer for $\Lambda$ production and in Fig. 3 for $\bar{\Lambda}$ production. The three curves represent the predictions from three possible scenarios proposed in Ref. [8] in the framework of the pQCD based model and the use of the reciprocity relation Eq.(5). The dotted curves correspond to pure valence quark contributions and for the dashed curves one has introduced a symmetric quark-antiquark sea, whereas it is asymmetric for the solid curves. Although this last case seems slightly favored, the simultaneous description of $\Lambda$ and $\bar{\Lambda}$ spin transfers remains hard to achieve and therefore more accurate data are badly needed (for a detailed discussion on this point, see Ref.[8] ).

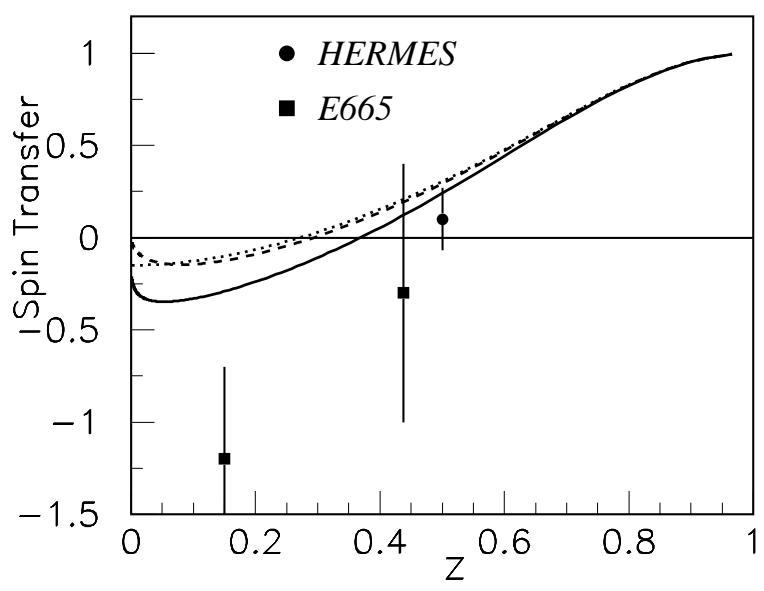

Figure 2. Three different predictions for the $z$ dependence of the $\Lambda$ spin transfer in charged lepton DIS, compared with data (Taken from Ref.[8]).

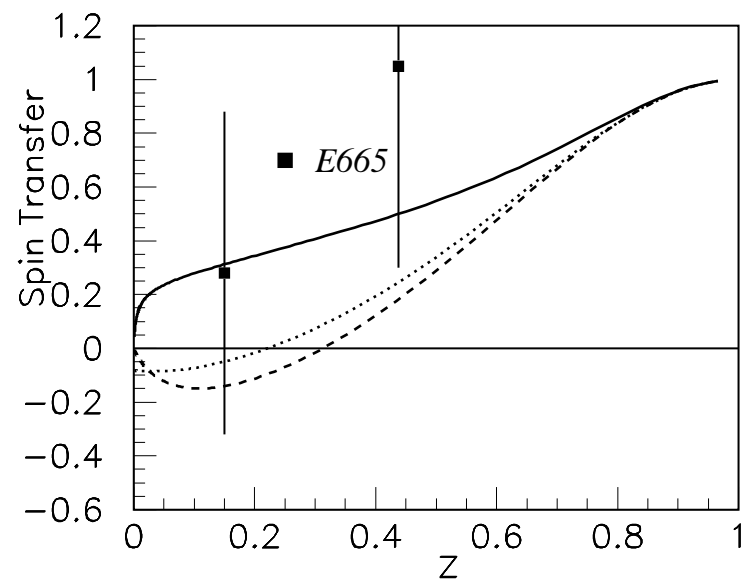

Figure 3. Three different predictions for the $z$ dependence of the $\bar{\Lambda}$ spin transfer in charged lepton DIS, compared with data (Taken from Ref.[8]). 


\section{Neutrino DIS process}

The main observation is based on the fact that neutrinos (antineutrinos) can be regarded as a purely polarized lepton beam since neutrinos are left-handed (antineutrinos are right-handed). Therefore they only interact with quarks of specific helicities and flavors. The scattering of a neutrino (antineutrino) beam on a hadronic target provides a source of polarized quarks with specific flavor. This important property makes neutrino (antineutrino) DIS, an ideal laboratory to study the flavor dependence of quark fragmentation.

If one measures, $\Lambda$ and $\bar{\Lambda}$ production in neutrino and antineutrino DIS, one can show [9] that the four longitudinal polarizations $P_{\nu}^{\Lambda}, P_{\bar{\nu}}^{\Lambda}, P_{\nu}^{\bar{\Lambda}}$ and $P_{\bar{\nu}}^{\bar{\Lambda}}$ can be expressed in terms of four fragmentation functions, allowing a clean separation between quarks and antiquarks. In Fig.4 we show the NOMAD data for $P_{\nu}^{\Lambda}$, compared with some model calculations from Ref.[7], as well as the predictions for the remaining three processes. Again more data are requiered to test these models but fortunately, one can expect future experimental possibilities from charged currents $e^{ \pm} p$ DIS, with HERA at DESY, CEBAF at Jefferson Lab. and eRHIC at BNL.

\section{Hyperons in $p p$ collisions}

Let us now move to another area where we can test these polarized fragmentation functions, by studying the spin transfer parameters in $p p$ collisions. A new polarized $p p$ collider at RHICBNL, which starts operating now, will allow to undertake a vast spin physics programme at high center-of-mass energies, up to $\sqrt{s}=500 \mathrm{GeV}$. First, we are interested in the reaction $\vec{p} p \rightarrow$ $\vec{\Lambda} X$, where both the initial proton and the produced $\Lambda$ are longitudinaly polarized and we consider the helicity transfer parameter $D_{L L}^{\Lambda}$ defined as follows

$D_{L L}^{\Lambda}\left(\eta, p_{T}\right)=\left(d \sigma_{++}-d \sigma_{+-}\right) /\left(d \sigma_{++}+d \sigma_{+-}\right)$,

where $\eta$ and $p_{T}$ are the rapidity and the transverse momentum of the outgoing $\Lambda . d \sigma_{++}\left[d \sigma_{+-}\right]$ denotes the cross section where the proton and
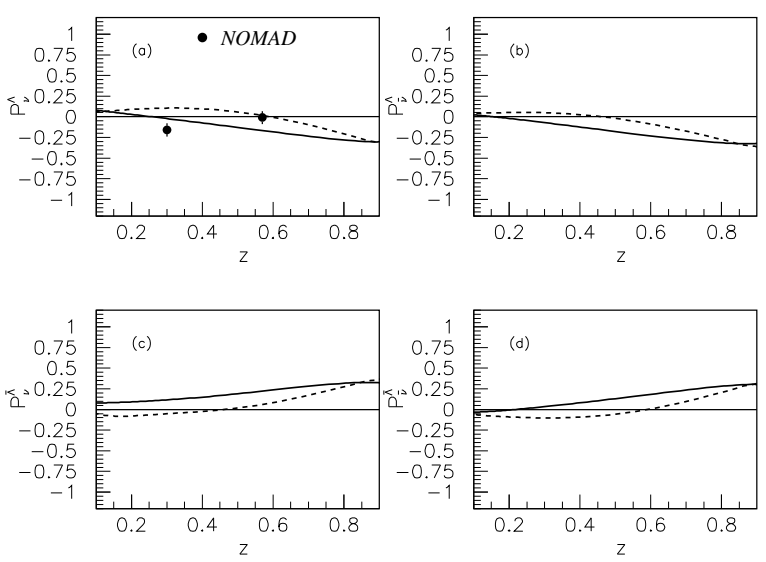

Figure 4. Two different predictions for the $z$ dependence of the $\Lambda$ and $\bar{\Lambda}$ polarization in neutrino and antineutrino DIS (Taken from Ref.|[7]).

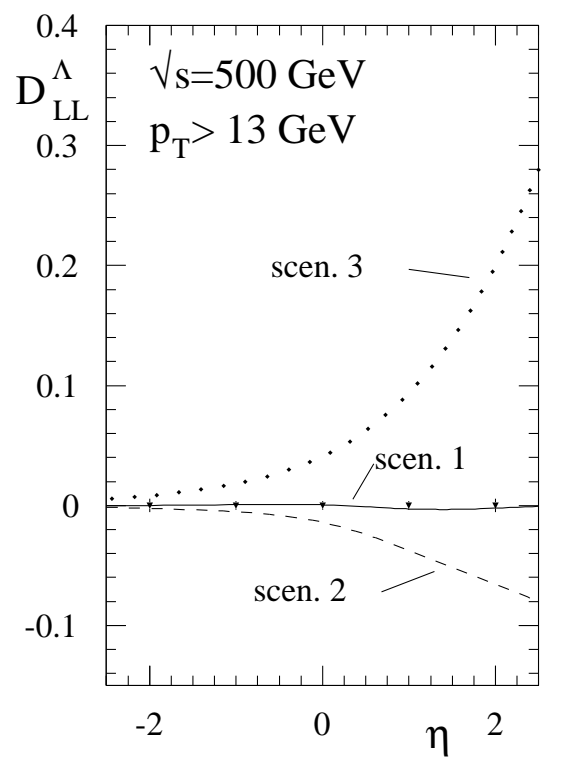

Figure 5. The helicity transfer parameter $D_{L L}^{\Lambda}$, versus the rapidity of the $\Lambda$ at RHIC-BNL maximum energy, using three different scenarios described in Section 2 (Taken from Ref.[10]). 

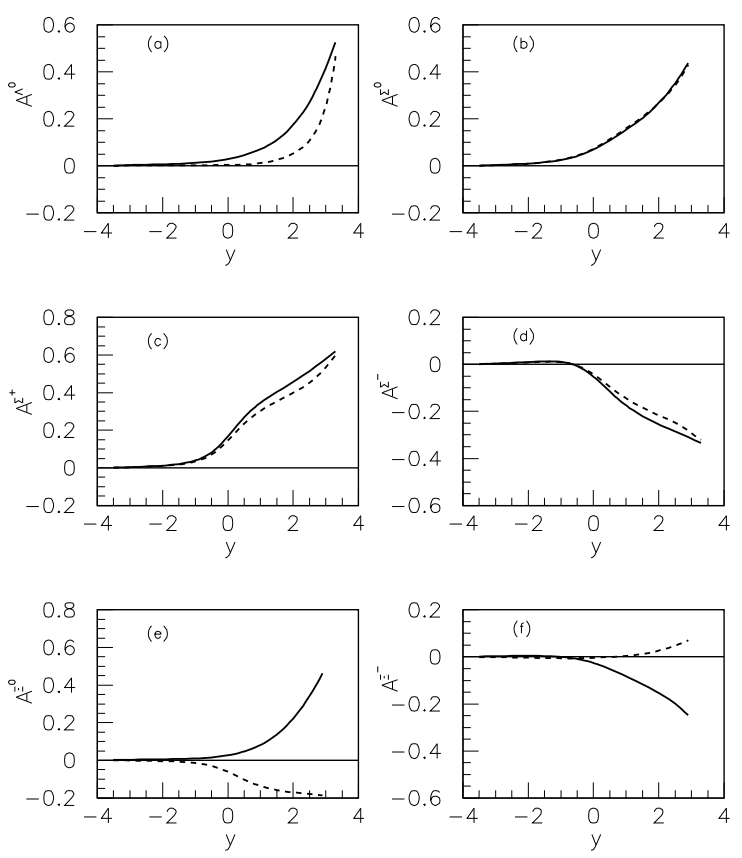

Figure 6. The helicity transfers, denoted here $A^{B}$, as functions of rapidity $y$ of the produced $\Lambda, \Sigma^{ \pm, 0}, \Xi^{-, 0}$, octet baryon members, in $\overrightarrow{p p}$ collisions at $\sqrt{s}=500 \mathrm{GeV}$, with the spin-dependent fragmentation functions in the pQCD counting rules analysis (solid curves) and the SU(6) quarkdiquark spectator model (dashed curves). Note that the dashed and solid curves in (b) almost overlap ( Taken from Ref. 11].

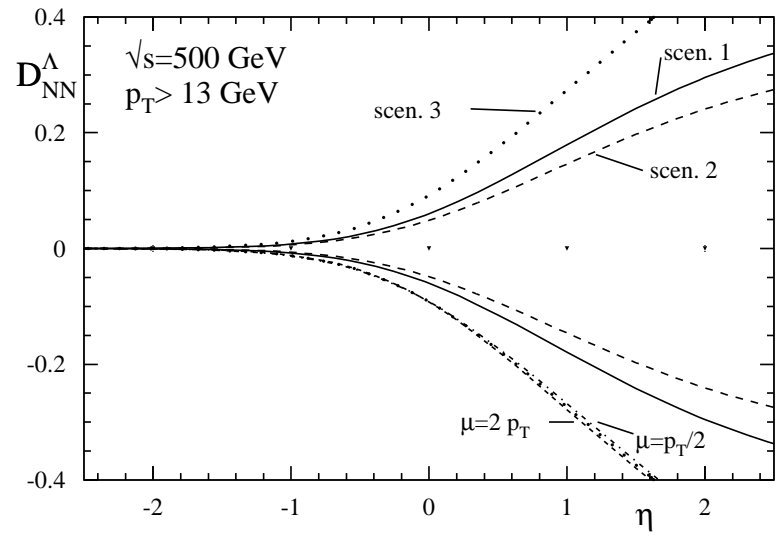

Figure 7. Upper and lower bounds for the spin transfer parameter $D_{N N}^{\Lambda}$, versus the rapidity of the $\Lambda$ at RHIC-BNL maximum energy. The small statistical errors are shown along the $\eta=0$ axis (Taken from Ref.|13]) .

the $\Lambda$ helicities have the same [opposite] sign. When $\eta$ is positive the $\Lambda$ has the direction of $\vec{p}$ and $p_{T}$ is always assumed to be large. $D_{L L}^{\Lambda}$ is directly related to the $\Delta D_{f}^{\Lambda}$ 's, whose exact expression can be found in Ref. [10], and therefore it is possible to make predictions, which are shown in Fig.5. Again one can see that the sign and magnitude of $D_{L L}^{\Lambda}$ are strongly correlated to the values of $N_{u}$ and given the smallness of the expected statistical errors, indicated in the figure along the horizontal axis, it will be now rather easy to pin down the right underlying mechanism. One notices that in all cases $D_{L L}^{\Lambda}$ is small for negative rapidity. This is due to the fact that the dominant subprocess is $g q \rightarrow g q$ and we have assumed that $\Delta D_{g}^{\Lambda}=0$. This feature is rather general and it was discussed in more details in Ref. 11]. It characterizes also other helicity transfers as shown in Fig.6, where we display 
the predicted helicity transfers for all the hyperons $\Lambda, \Sigma^{ \pm, 0}, \Xi^{-, 0}$, resulting from two models of the fragmentation functions.

Finally, we can also consider the case where both the proton and the $\Lambda$ are transversely polarized and the corresponding spin transfer parameter $D_{N N}^{\Lambda}$, is defined as in Eq.(7), where helicity is replaced by transverse polarization. This spin observable is related to the quark transversity distributions, so called $h_{1}^{q}\left(x, Q^{2}\right)$ and to the transversity fragmentation functions $\Delta_{T} D_{f}^{\Lambda}\left(z, Q^{2}\right)$ mentioned in Section 2. Needless to say that we have no experimental information on these quantities. However we can derive some bounds on $D_{N N}^{\Lambda}$, using positivity arguments. We recall that positivity leads to the inequality 12

$2\left|h_{1}^{q}\left(x, Q^{2}\right)\right| \leq q\left(x, Q^{2}\right)+\Delta q\left(x, Q^{2}\right)$,

whose validity has been established up to NLO QCD corrections. A similar constraint holds for hadron fragmentation functions 13], namely

$2\left|\Delta_{T} D_{q}^{H}\left(z, Q^{2}\right)\right| \leq D_{q}^{H}\left(z, Q^{2}\right)+\Delta D_{q}^{H}\left(z, Q^{2}\right)$.

By saturating the above inequalities, we get an estimate for an upper and lower bounds for $D_{N N}^{\Lambda}$ which are displayed in Fig.7. The larger allowed region corresponds to scenario 3), mentioned earlier, due to the value of $N_{u}$ and from the expected statistical accuracy, we get rather small error bars.

To summarize, we have now some knowledge of the unpolarized fragmentation functions $D_{f}^{\Lambda}\left(z, Q^{2}\right)$, but we miss a better flavor separation, which can be properly achieved by means of neutrino (antineutrino) DIS or charged currents in $e^{ \pm} p$ DIS. The corresponding polarized fragmentation functions $\Delta D_{f}^{\Lambda}\left(z, Q^{2}\right)$ are poorly known and more data is needed for testing several theoretical models proposed in the literature. Future measurements of $\Lambda$ and $\bar{\Lambda}$ production in semiinclusive charged lepton DIS will improve the situation and this can be generalized to the case of the production of the other hyperons $\Sigma^{ \pm, 0}, \Xi^{-, 0}$. The new polarized $p p$ collider at RHIC-BNL, with high luminosity and high energy, is also a very powerful facility to study unpolarized and polar- ized fragmentation functions for all octet baryon members.

Acknowledgements

It is my pleasure to thank the orginazers S.D.Bass, A. De Roeck and A. Deshpande for their invitation and for setting up this excellent workshop in such pleasant and stimulating atmosphere.

\section{REFERENCES}

1. D. de Florian, M. Stratmann and W. Vogelsang, Phys. Rev. D57 (1998) 5811.

2. C. Boros, J.T. Londergan and A.W. Thomas, Phys. Rev. D62 (2000) 014021.

3. M. Burkardt and R.L. Jaffe, Phys. Rev. Lett. 71 (1993) 2537.

4. A. Kotzinian, A. Bravar and D. von Harrach, Eur. Phys. J. C2 (1998) 329.

5. B.-Q. Ma, I. Schmidt and J.J. Yang, Phys. Rev. D61 (2000) 034017.

6. V.N. Gribov and L.N. Lipatov, Phys. Lett. B37 (1971) 78 ; Sov. J. Nucl. Phys. 15 (1972) 675.

7. B.-Q. Ma, I. Schmidt, J. Soffer and J.J. Yang, hep-ph/0110029, Phys. Rev. D (to appear).

8. B.-Q. Ma, I. Schmidt, J. Soffer and J.J. Yang, Euro. Phys. J. C16 (2000) 657.

9. B.- Q. Ma and J. Soffer, Phys. Rev. Lett. 82 (1999) 2250.

10. D. de Florian, M. Stratmann and W. Vogelsang, Phys. Rev. Lett. 81 (1998) 530.

11. B.-Q. Ma, I. Schmidt, J. Soffer and J.J. Yang, hep-ph/0107157, Nucl. Phys. A (to appear).

12. J. Soffer, Phys. Rev. Lett. 74 (1995) 1292; W. Vogelsang, Phys. Rev. D57 (1998) 1886; C. Bourrely, J. Soffer and O.V. Teryaev, Phys. Lett. B420 (1998) 1886.

13. D. de Florian, J. Soffer, M. Stratmann and W. Vogelsang, Phys.Lett. B439 (1998) 176. 